\title{
Studying ICD-11 Primary Health Care bodily stress syndrome in Brazil: do many functional disorders represent just one syndrome?
}

\author{
Sandra Fortes, ${ }^{1}$ iD Carolina Ziebold, ${ }^{2}$ Geoffrey M. Reed, ${ }^{3,4}$ Rebeca Robles-Garcia, ${ }^{5}$ Monica R.
} Campos, ${ }^{6}$ Emilene Reisdorfer, ${ }^{7}$ Ricardo Prado, ${ }^{2}$ David Goldberg, ${ }^{8}$ Linda Gask, ${ }^{9}$ Jair J. Mari ${ }^{2}$ iD

${ }^{1}$ Faculdade de Ciências Médicas, Universidade do Estado do Rio de Janeiro (UERJ), Rio de Janeiro, RJ, Brazil. ${ }^{2}$ Departamento de Psiquiatria, Universidade Federal de São Paulo (UNIFESP), São Paulo, SP, Brazil. ${ }^{3}$ World Health Organization (WHO), Geneva, Switzerland. ${ }^{4}$ Global Mental Health Program, Columbia University Medical Center, New York, NY, USA. ${ }^{5}$ Instituto Nacional de Psiquiatría Ramón de la Fuente Muñiz, Ciudad de México, CDMX, Mexico. ${ }^{6}$ Escola Nacional de Saúde Pública Sergio Arouca (ENSP), Fundação Oswaldo Cruz (Fiocruz), Rio de Janeiro, RJ, Brazil. ${ }^{7}$ Center for Addition and Mental Health, Toronto, Ontario, Canada. ${ }^{8}$ Institute of Psychiatry, King's College London, Institute of Psychiatry, King's College London, United Kingdom. ${ }^{9}$ University of Manchester, Manchester, United Kingdom. iD SF https://orcid. org/0000-0002-9918-1555, (iD JJM https://orcid.org/0000-0002-5403-0112

\begin{abstract}
Objective: Disorders characterized by "distressing unexplained somatic symptoms" are challenging. In the ICD-11 Primary Health Care (PHC) Guidelines for Diagnosis and Management of Mental Disorders (ICD-11 PHC), a new category, bodily stress syndrome (BSS), was included to diagnose patients presenting unexplained somatic symptoms. The present study investigated the association of BSS with anxiety, depression, and four subgroups of physical symptoms in a Brazilian primary health care (PHC) sample. Methodology: As part of the international ICD-11 PHC study, 338 patients were evaluated by their primary care physicians, followed by testing with Clinical Interview Schedule (CIS-R) and World Health Organization Disability Assessment Schedule, Version 2.0 (WHODAS 2.0). BSS was diagnosed in the presence of at least three somatic symptoms associated with incapacity. The association between anxiety, depression, and four subgroups of physical symptoms with being a BSS case was analyzed. Results: The number of somatic symptoms was high in the overall sample of 338 patients (mean = 8.4), but even higher in the 131 BSS patients (10.2; $p<0.001)$. Most BSS patients $(57.3 \%)$ had at least three symptoms from two, three, or four subgroups, and these were associated with anxiety and depression in $80.9 \%$ of these patients. The symptom subgroup most strongly associated with "being a BSS" case was the non-specific group $(\mathrm{OR}=6.51$; $95 \% \mathrm{Cl} 1.65-24.34)$, followed by musculoskeletal (OR $=2,31 ; 95 \% \mathrm{Cl} 1.19-4.72)$.

Conclusion: Somatic symptoms were frequent in a sample of PHC patients in Brazil. In the present sample, one third were BSS cases and met the criteria for at least two symptom subgroups, supporting the hypothesis that different functional symptoms are related to each other.
\end{abstract}

Keywords: Community mental health; stress; diagnosis and classification; somatoform disorders; bodily distress syndrome

\section{Introduction}

Somatic symptoms that are not explained by somatic diseases, traditionally called "medically unexplained somatic symptoms," are among the most controversial/ challenging facets of mental health. Each disease classification system, including the ICD-9, the ICD-10 and its Primary Health Care (PHC) Guidelines for Diagnosis and Management of Mental Disorders (ICD-10 PHC), and all DSM versions (from DSM-III to the current DSM-5), employs different labels when referring to unexplained symptoms: somatization, conversion, and functional syndromes. ${ }^{1-5}$

Correspondence: Sandra Fortes, Rua Barão de Mesquita, 36/401, Maracanã, CEP 20540-006, Rio de Janeiro, RJ, Brazil.

E-mail: sandrafortes@gmail.com

Submitted Oct 07 2017, accepted Jan 29 2018, Epub Oct 112018.
Specialists in this field have underscored the urgent need to improve definitions so that better therapeutic alternatives can be developed for these patients. ${ }^{6-9}$

In the last century, patients with medically unexplained somatic symptoms were referred to as having "somatization"; however, there are several problems that make it difficult to define precisely which pathological processes are involved in this concept. ${ }^{10-12}$ The first difficulty is the fact that the presence of somatic symptoms that are not explained by physical disorders is quite frequent, and may be a normal process. Others have suggested these symptoms as idioms of distress or cultural patterns of

How to cite this article: Fortes S, Ziebold C, Reed GM, Robles-Garcia R, Campos MR, Reisdorfer E, et al. Studying ICD-11 Primary Health Care bodily stress syndrome in Brazil: do many functional disorders represent just one syndrome? Braz $\mathrm{J}$ Psychiatry. 2019;41:15-21. http://dx.doi.org/10.1590/1516-44462018-0003 
expressing emotional suffering. ${ }^{13}$ According to Desjarlais et al., ${ }^{14}$ for example, cultural background influences whether depression will be expressed in psychological, emotional, or physical terms. However, somatic symptoms may become chronic and associated with elevated degrees of disability, usually involving abnormal illness behavior. ${ }^{15-17}$ Furthermore, somatic symptoms are often strongly associated with common mental disorders such as anxiety and depression syndromes, ${ }^{18-21}$ and also with a variety of conditions ranging from conversion disorder (described since Ancient Greece as hysteria) to modern functional syndromes, including chronic fatigue, fibromyalgia, and irritable bowel syndrome, where a significant overlap of symptoms can be found. ${ }^{22}$ Indeed, several authors have raised the hypothesis that functional syndromes may reflect different aspects of a single pathological process, rather than independent syndromes. ${ }^{23,24}$

To explain the pathological process in patients with somatic symptoms in the absence of clinical disease, Fink et al. ${ }^{25-28}$ have proposed the notion of a bodily distress syndrome (BDS), emphasizing the co-occurrence of cardiopulmonary, musculoskeletal, and gastrointestinal symptom clusters. The authors explicitly link these symptom clusters to "functional" syndromes of non-cardiac chest pain, fibromyalgia, and irritable bowel syndrome. They also include an additional cluster of "general" or "non-specific" symptoms (e.g., concentration difficulties, memory disability, fatigue, dizziness, and headache), theorized as also corresponding to chronic fatigue syndrome. This structural model has been tested, supporting the notion that underlying common features (a general factor) unite distinct clusters of somatic symptoms.

The World Health Organization (WHO) is currently developing the ICD-11. Since the ICD-10, the WHO has introduced a separate classification for mental disorders encountered in PHC settings, the ICD-10 PHC; thus, a special WHO Working Group was established to review the ICD-10 PHC, and three new mental disorder diagnostic categories were proposed for inclusion in ICD-11 $\mathrm{PHC}^{29-31}$ The first one involves a unified diagnosis of anxiety and depression as "anxious depression,"30 supporting that the co-occurrence of these conditions in $\mathrm{PHC}$ is the rule rather than the exception; the second is BSS, ${ }^{31}$ which refers to patients with somatic symptoms, with or without health anxiety, which is the third category involved, covering patients with excessive worries about their health. BSS, the focus of the present study, is defined as characterized by four aspects: complaints of three or more persistent somatic symptoms that are distressing, result in significantly disability, interfere with daily functioning, and are not caused by a known physical pathology. These somatic symptoms may coincide with the symptoms included in any of the four clusters of symptoms originally proposed by Fink et al., or else with the symptoms described in any other system, as originally proposed in the category defined as "multisystem BDS" by Fink et al. ${ }^{25-28}$

The first results of the field study to analyze BSS, performed in five countries (Spain, China, Pakistan, Mexico and Brazil), have already been published. ${ }^{31} \mathrm{An}$ important question addressed was the overlap between
BSS and depressive and anxiety symptoms. However, the occurrence of the symptom subgroups as proposed in the Danish studies by Fink et al. ${ }^{26-28}$ was also assessed in order to determine whether these clusters would be a useful basis for BSS in ICD-11 PHC. The results raised some interesting issues: 1) all patients presented a high number of somatic symptoms (mean of 10.5 [standard deviation = 5.3]), and, with the exception of the Chinese sample, most patients presented symptoms from more than one symptom subgroup, with the most common pattern being three or more symptoms in multiple subgroups $(57.9 \%)$; 2) an important association of BSS with anxiety and depression was confirmed in $78.9 \%$ of the total sample, with different distributions among the countries (with China having the lower rate of co-occurrence = 45.5\%). However, the fact that not all patients with BSS presented anxiety and/or depression suggested that BSS is not necessarily contingent on the presence of depression/anxiety.

In the present study, we examined how the four BSS symptom subgroups relate to the presence of the overall syndrome (BSS), to one another, and to anxiety and depressive disorders in a Brazilian sample. A secondary aim was to examine whether there was any other significant physical symptom, different from the four groups already defined, associated with BSS in the Brazilian sample.

\section{Methods}

\section{Participants}

This cross-sectional study was designed to test aspects of the proposed ICD-11 PHC that represent substantial changes from the ICD-10 PHC. The study involved the evaluation of two groups of PHC patients: 1) patients identified as psychologically distressed by primary care physicians (PCPs) and evaluated according to the proposed ICD-11 PHC diagnostic scheme for depression and anxiety, including the new diagnosis of anxious depression $^{30}$; and 2) adult patients in whom PCPs detected at least three distressing somatic symptoms or substantial anxiety about their health status, not fully explained by an identified medical condition. These patients were evaluated according to the proposed ICD-11 PHC diagnostic scheme for BSS and health anxiety. The cooccurrence of BSS and health anxiety with depression and anxiety was also evaluated. ${ }^{31}$

Data included in the present analyses were collected in the cities of São Paulo and Rio de Janeiro, Brazil, from October 2013 to July 2015. Participants were adults (at least 18 years of age) who came for primary care consultations as a part of their usual care in Unified Health System (SUS) primary care clinics in Rio and São Paulo selected for their interest in participating along with availability of local resources and infrastructure (i.e., room for patient interview). For both groups, PCPs were asked not to refer patients with severe acute physical illness or pain or who would have difficulty understanding the interview questions for reasons of language proficiency. As described in the original articles, ${ }^{30,31}$ PCPs were provided 
with a single half-day training session on the three new categories evaluated in the overall study (anxious depression, BSS, and health anxiety), using slides prepared by the WHO, including discussion among the professionals and evaluation of cases prepared in advance.

For patients presenting at least three distressing somatic symptoms or substantial anxiety about their health status, and agreeing to participate in the study, the PCPs completed an Encounter Form containing detailed information about the number and type of somatic symptoms currently experienced, including a list of 29 somatic symptoms based on the symptoms used by Fink et al. ${ }^{27,28}$ The Encounter Form also included information about all other proposed diagnostic requirements of both BSS and health anxiety, including distress and disability. Patients who agreed to participate underwent a structured diagnostic interview, the revised Clinical Interview Schedule (CIS-R), ${ }^{32}$ administered by research assistants who underwent an initial training lasting two days at the Universidade Federal de São Paulo under the guidance of one of the authors (RP) followed by supervised application of the instrument in at least five patients. During the entire field trial, research assistants had weekly meetings with the supervisors (RP and SF) to discuss problems and solve doubts.

Research assistants also administered the 12-item version of the WHO Disability Assessment Schedule, Version 2.0 (WHODAS 2.0). ${ }^{33} \mathrm{CIS}-\mathrm{R}$ diagnostic requirements for diagnoses of common mental disorders were applied and then modified as proposed by the ICD-11 PHC. One of the main modifications was the inclusion of the separate category of anxious depression, in which the requirements for both depression and current anxiety consider a duration of 2 weeks rather than several months. The Brazilian Field Trial included initially 55 patients as part of the international study. However, in order to better analyze anxious depression in a Brazilian population, the field study was continued until March 2015.

All procedures were approved by the WHO research ethics review committee and by the appropriate local institutional review bodies (in Brazil: CAAE 22689113.4. 1001.5259).

\section{Data analysis}

Data were analyzed using SPSS version 22. Descriptive analyses included frequencies and proportions of socialdemographic characteristics, number of somatic cluster overlap (number of clusters in which participants presented three or more somatic symptoms), and anxiety and depression comorbidity (other ICD-11 PHC diagnoses). Between-groups differences in frequencies (proportions) were tested using two-tailed chi-square $\left(\chi^{2}\right)$ analysis with significance at $p<0.05$. Odds ratios (OR) were calculated with $95 \%$ confidence intervals $(95 \% \mathrm{Cl})$. For ordinal variables, the mean and standard deviation were calculated. Two-tailed one-way analyses of variance (ANOVA) were carried out to test whether there was an overall difference in disability levels (using mean scores on 12-WHODAS 2.0) associated with anxious depression comorbidity vs. somatic symptom subgroup overlap.
Pairwise comparisons among values where the overall test reached a significance level of $p<0.05$ were corrected for multiple family-wise comparisons using the Bonferroni correction and a parameter of significance of $\mathrm{p}<0.05$.

\section{Results}

PCPs initiated 347 somatic symptom screenings. Of these, information was missing in six. Three patients refused to participate because they lacked time or did not feel well enough. Thus, 338 patients were screened. Of these, only 131 fulfilled BSS criteria (Figure 1). They were mostly women $(n=113 ; 86 \%)$, with mean age of $47.7 \pm$ 13.7 years; $34 \%(n=45)$ worked full time, $18 \%(n=24)$ worked part time, $16 \%(n=21)$ were retired, $22 \%(n=29)$ were housemakers, and $4 \%(n=5)$ were unemployed due to illness.

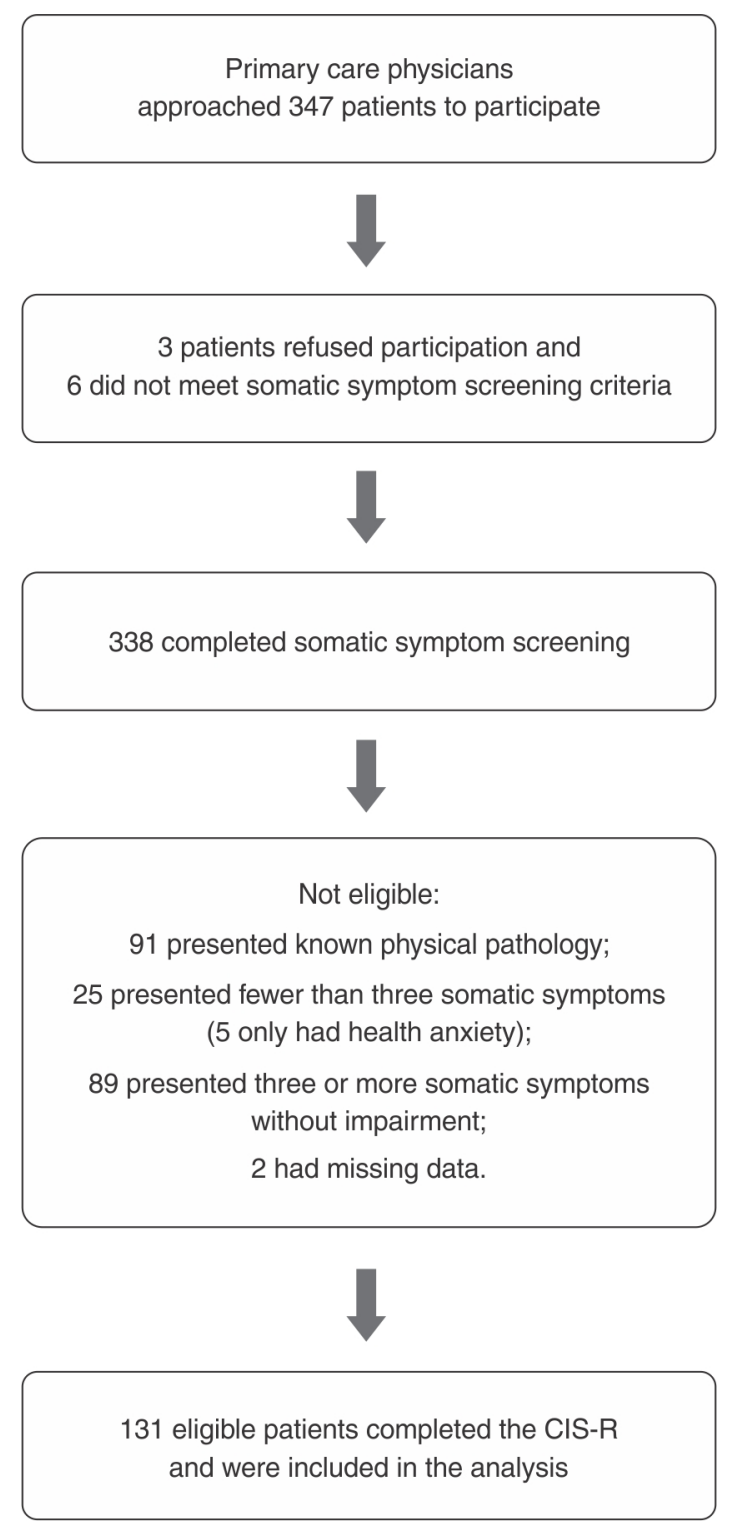

Figure 1 Study flow chart. CIS-R = Clinical Interview Schedule. 
The number of somatic symptoms was high among the overall group of 338 patients, including those who were not considered as having BSS: $8.2 \pm 5.6$ symptoms. Nevertheless, the number of symptoms was higher in 131 patients meeting the criteria for BSS: 10.2 \pm 4.9 (statistically significant difference between patients with or without BSS according to $t$ test; $t=3.406$, degrees of freedom $=344, p<0.001$ ). Most patients with BSS had at least three symptoms from the musculoskeletal subgroup $(66.4 \%)$, followed by non-specific symptoms $(48.1 \%)$ and cardiovascular symptoms (43.5\%). Gastrointestinal symptoms were present in $34.4 \%$ of these patients.

In our study, only $17.6 \%(n=23)$ of the patients with BSS did not present another common mental disorder at the time of evaluation. Among the $81 \%$ who had another mental disorder, anxious depression was the most common, detected in $52 \%$ of the cases $(n=68)$, followed by current anxiety $(20.6 \%, n=27)$. There were also eight cases of depression and three of sub threshold anxious depression. Data were missing in two cases. Anxious depression was the category most strongly associated with disability (Table 1).

Considering the four subgroups of symptoms studied (symptoms associated with non-cardiac chest pain, fibromyalgia, irritable bowel syndrome, and chronic fatigue syndrome), most of the patients with BSS $(57.3 \%)$ presented at least three symptoms in two, three, or four of these subgroups, confirming a significant overlap in this Brazilian sample. We found that in $16 \%$ of the patients who had at least three symptoms, they were spread across different subgroups, showing a diffuse pattern; $26.7 \%$ presented symptoms in only one specific subgroup.
The level of disability measured by 12-WHODAS increased with the number of subgroups from which the patients had symptoms. Having widespread symptoms was associated with a level of disability similar to that of having symptoms from only one or two groups (Table 2). However, disability was significantly greater $(p=0.045)$ if the person had symptoms from four subgroups (OR $13.09,95 \% \mathrm{Cl} 9.85-16.33$ ) than for only one group (OR $6.86,95 \% \mathrm{Cl} 4.35-9.37)$.

However, the association between symptoms from these different subgroups and being a BSS case differed considerably. The group of symptoms most strongly associated with "being a BSS case" was the non-specific category $(\mathrm{OR}=6.51,95 \% \mathrm{Cl} 1.69-25.05)$, followed by musculoskeletal subgroup $(\mathrm{OR}=2.31,95 \% \mathrm{Cl} 1.17-4.58)$. The presence of gastrointestinal symptoms was not significantly $(p=0.093)$ associated with being a case of BSS in the Brazilian sample (Table 3 ).

Finally, the possibility that other types of unexplained symptoms could be present, in addition to those included in the four subgroups studied, was considered. Only $10 \%$ of the patients presented other medically unexplained symptoms that were not in the four subgroups. These were various symptoms that occurred individually, such as abdominal pain, thoracic pain, somnolence, flatulence, and genital-urinary symptom.

\section{Discussion}

In the present sample, most BSS patients presented symptoms from three or four subgroups (symptom subgroups associated with the most common functional disorders - cardiovascular, irritable bowel, fibromyalgia and chronic fatigue syndrome), thus supporting the idea

Table 1 Distribution of ICD-11 PHC diagnoses by levels of World Health Organization Disability Assessment Schedule (WHODAS) disability

\begin{tabular}{lcc}
\hline Diagnosis & $\mathrm{n}$ & Disability, mean (95\%Cl) \\
\hline Anxious depression & 68 & $11.69(9.55-13.84)$ \\
Subthreshold anxious depression & 3 & $7.33(-0.65-15.32)$ \\
Current anxiety & 27 & $7.63(4.88-10.38)$ \\
Depressive episode & 8 & $6.00(0.89-11.11)$ \\
No diagnosis identified & 23 & $3.61(1.66-5.56)^{*}$ \\
Total & 129 & $8.95(7.52-10.37)$ \\
\hline
\end{tabular}

$95 \% \mathrm{Cl}=95 \%$ confidence interval.

* Significant lower than anxious depression $(p<0.001)$. ANOVA $F_{4.124}: 5.328 . p<0.001$

Table 2 Level of disability in World Health Organization Disability Assessment Schedule (WHODAS) according to the number of subgroups with at least three symptoms presented by the patients

\begin{tabular}{|c|c|c|c|}
\hline Number of groups* by BSS & $\mathrm{n}$ & Prevalence (\%) & 12-WHODAS, mean $(95 \% \mathrm{Cl})^{\dagger}$ \\
\hline Only diffuse symptoms & 21 & 16.0 & $8.43(4.24-12.62)$ \\
\hline One subgroup & 35 & 26.7 & $6.86(4.35-9.37)$ \\
\hline Two subgroups & 31 & 23.7 & $8.41(4.95-11.88)$ \\
\hline Three subgroups & 21 & 16.0 & $9.14(6.09-12.19)$ \\
\hline Four subgroups & 23 & 17.6 & $13.09(9.85-16.33)$ \\
\hline Total & 131 & 100.0 & $8.95(7.52-10.37)$ \\
\hline
\end{tabular}

$95 \% \mathrm{Cl}=95 \%$ confidence interval; BSS = bodily stress syndrome.

* Groups: cardiopulmonary, musculoskeletal, gastrointestinal and general symptoms.

ANOVA $=F_{4,124}=2.18, p=0.075$.

$\ddagger_{\mathrm{n}}=29$, two missing data. 
Table 3 Association of different types of symptoms (presence of at least one symptom from different subgroups) with being a BSS case

\begin{tabular}{|c|c|c|c|c|c|}
\hline \multirow{2}{*}{ Symptoms } & \multicolumn{3}{|c|}{ Bodily distress syndrome } & \multirow{2}{*}{$p$-value $\left(\chi^{2}\right)^{*}$} & \multirow{2}{*}{ OR $(95 \% \mathrm{Cl})$} \\
\hline & Yes, n (\%) & No, n (\%) & Total, n (\%) & & \\
\hline \multicolumn{6}{|c|}{ Gastrointestinal symptoms } \\
\hline Yes & $97(74.0)$ & $135(65.2)$ & $232(68.6)$ & 0.093 & $1.30(0.95-1.79)$ \\
\hline No & $34(26.0)$ & $72(34.8)$ & $106(31.4)$ & & \\
\hline \multicolumn{6}{|c|}{ Musculoskeletal symptoms } \\
\hline Yes & $124(94.7)$ & $175(84.5)$ & $299(88.5)$ & 0.005 & $2.31(1.17-4.58)$ \\
\hline No & $7(5.3)$ & $32(15.5)$ & $39(11.5)$ & & \\
\hline \multicolumn{6}{|c|}{ Cardiovascular symptoms } \\
\hline Yes & $107(81.7)$ & $139(67.1)$ & $246(72.8)$ & 0.004 & $1.67(1.15-2.42)$ \\
\hline No & $24(18.3)$ & 68 (32.9) & $92(27.2)$ & & \\
\hline \multicolumn{6}{|c|}{ Non-specific symptoms } \\
\hline Yes & $129(98.5)$ & $178(86.0)$ & $307(90.8)$ & $<0.001$ & $6.51(1.69-25.05)$ \\
\hline No & $2(1.5)$ & $29(14.0)$ & $31(9.2)$ & & \\
\hline
\end{tabular}

$95 \% \mathrm{Cl}=95 \%$ confidence interval; OR $=$ odds ratio.

$*$ Pearson chi-square values (degrees of freedom $=1$ ): gastrointestinal system $=2.905$; musculoskeletal system $=8.042$; cardiovascular system $=8.550 ;$ non-specific symptoms $=15.007$.

that these syndromes may represent diverse aspects of a single disorder. The high comorbidity of BSS with common mental disorders was again confirmed, as only $20 \%$ of the patients did not present any subtype of anxiety or depression. However, in this Brazilian sample of BSS patients from a PHC setting, the most common disorders were anxious depression and anxiety, with only a minority presenting depression. Physical symptoms are an important aspect of common mental disorders in Brazil. In our sample, the mean number of somatic symptoms in all patients was 8.4 , a figure that increased to 10.2 among individuals with BSS. Physical symptoms are well known as part of a Latin American cultural pattern of emotional distress, anxiety, and depression, ${ }^{34-36}$ and Brazil is clearly no exception. However, for the diagnosis of BSS, physical symptoms are specifically considered in association with disability. So, even though BSS is strongly associated with anxiety and depressive syndromes, we have confirmed that in this Brazilian sample (especially with anxious depression, the new syndrome included in ICD-11 PHC, and current anxiety), BSS cannot be considered to involve the exact same processes as anxiety and depression, since almost $20 \%$ of the BSS patients did not present any syndrome related to anxiety or depression. This supports the proposal of the WHO Working Group regarding the inclusion of BSS as a new category in the ICD11 PHC. $^{37}$

Another important result was that most patients (57.3\%) fulfilled criteria from two, three, or four symptom subgroups; $16 \%$ had symptoms scattered in all subgroups, and $26.7 \%$ had symptoms from only one subgroup. These results demonstrate the overlap among subgroups and support the idea that the different functional syndromes may be part of one general syndrome instead of being independent disorders. ${ }^{23}$ Among the four subgroups, only the gastrointestinal symptom subgroup was not significantly associated with BSS in this Brazilian sample. The non-specific symptom cluster was the most strongly associated with being a BSS case.
Disability was more strongly associated with the presence of anxious depression and with a greater number of systems involved, which may indicate an increasing level of severity in BSS associated with a greater number of complaints in multiple systems.

Additionally, no other symptom not originally included in the four symptom subgroups was found to be significantly associated with the presence of BSS in this Brazilian sample. The range of somatic symptoms that is presented by distressed patients in primary care settings around the world is remarkably similar, including the physical symptoms associated with depression. ${ }^{38}$ Moreover, this finding weakens the importance of considering any other culture-specific somatic symptom for the definition of BSS in ICD-11 PHC.

The study has several limitations. The sample is not a large one and only patients from SUS units in the two largest cities in the country were examined. Brazil has a population of over $\mathbf{2 0 0}$ million and marked regional and socioeconomic differences. Additionally, the primary aim of the study was to test a new category from ICD-11 PHC, and the patients were selected as probable cases by their physicians. Therefore, this study investigated neither the prevalence of BSS in the Brazilian population nor the frequency of somatic symptoms in our general population. Our results underscore the importance of BSS for the daily routine of PCPs, as already discussed in previous papers, ${ }^{29,31}$ supporting its inclusion in the ICD-11 PHC. Somatic symptoms are extremely frequent, and their association with emotional distress an important aspect of primary mental health care. BSS as a syndrome is more adequate for the understanding of these patients than the Somatic Symptoms Syndrome described in DSM-5, ${ }^{39}$ as patients in primary care settings are not as severe or chronic as those attending specialized care. Another important contribution of our study relates to the understanding of the several functional syndromes that can be found in different subspecialties in medicine. Primary care patients are often only starting pathological processes. 
Being able to study this group will help us understand the development of these disorders.

Our study confirms that BSS, the new category proposed by the ICD-11 PHC classification, represents an adequate category to classify the disorders characterized by somatic symptoms not explained by a medical condition in a sample of the Brazilian population. An important finding was that most patients presented sufficient symptoms to fulfill criteria for two or more symptom subgroups in BSS, which are related to the most common functional syndromes, thus supporting the idea that these syndromes may represent different facets of a single pathological process. It is also important to notice that BSS is strongly associated with anxiety and depression, especially anxious depression. Further studies should be performed to investigate the association between different symptom subgroups and anxiety and depression, to shed light on the possible pathophysiological processes involved in the presence of somatic symptoms not explained by medical conditions.

\section{Acknowledgements}

JJM is a senior researcher at the National Research Council. The field study in Brazil was funded by Conselho Nacional de Desenvolvimento Científico e Tecnológico (CNPq 476905/2012-5). CZ is PhD candidate (CNPq scholarship) in the Graduate Program in Psychiatry, Universidade Federal de São Paulo, Brazil.

\section{Disclosure}

The authors report no conflicts of interest.

\section{References}

1 World Health Organization (WHO). International classification of disease. 9th revision. Geneva: WHO; 1977.

2 World Health Organization (WHO). The ICD-10 classification of mental and behavioural disorders: clinical descriptions and diagnostic guidelines [Internet]. 1992 [cited 2018 Feb 06]. www.who.int/classifi cations/icd/en/bluebook.pdf.

3 Ustün TB, Goldberg D, Cooper J, Simon GE, Sartorius N. New classification for mental disorders with management guidelines for use in primary care: ICD-10 PHC chapter five. $\mathrm{Br} \mathrm{J}$ Gen Pract. 1995;45:211-5.

4 American Psychiatric Association. Diagnostic and Statistical Manual of Mental Disorders, Third Revised Edition (DSM-III-R). Washington: American Psychiatric Association; 1987.

5 American Psychiatric Association. Diagnostic and Statistical Manual of Mental Disorders, Fourth Edition, Text Revision (DSM-IV-TR). Arlington: American Psychiatric Publishing; 2000.

6 Levenson $\mathrm{JL}$. A rose by any other name is still a rose. J Psychosom Res. 2006;60:325-6.

7 Mayou R, Kirmayer LJ, Simon G, Kroenke K, Sharpe M. Somatoform disorders: time for a new approach in DSM-V. Am J Psychiatry. 2005;162:847-55.

8 Sykes R. Somatoform disorders in DSM-IV: mental or physical disorders? J Psychosom Res. 2006;60:341-4.

9 Hiller W, Rief W. Why DSM-III was right to introduce the concept of somatoform disorders. Psychosomatics. 2005;46:105-8.

10 De Gucht V, Fischler B. Somatization: a critical review of conceptual and methodological issues. Psychosomatics. 2002;43:1-9.

11 Gureje O, Simon GE. The natural history of somatization in primary care. Psychol Med. 1999;29:669-76.

12 Lipowski ZJ. Somatization: the concept and its clinical application. Am J Psychiatry. 1988;145:1358-68.
13 Kirmayer LJ, Young A. Culture and somatization: clinical, epidemiological, and ethnographic perspectives. Psychosom Med. 1998;60: 420-30.

14 Desjarlais R. World mental health. Problems and priorities in lowincome countries. Oxford: Oxford University; 1996.

15 Thomassen R, Van Hemert AM, Huyse FJ, van der Mast RC, Hengeveld MW. Somatoform disorders in consultation-liaison psychiatry: a comparison with other mental disorders. Gen Hosp Psychiatry. 2003;25:8-13.

16 Smith GR Jr, Monson RA, Ray DC. Patients with multiple unexplained symptoms. Their characteristics, functional health and health care utilization. Arch Intern Med. 1986;146:69-72.

17 Spitzer RL, Kroenke K, Linzer M, Hahn SR, Williams JB, de Gruy FV 3rd, et al. Health-related quality of life in primary care patients with mental disorders. Results from the PRIME-MD 1000 study. JAMA. 1995;274:1511-7.

18 De Waal MW, Arnold IA, Eekhof JA, Van Hemert AM. Somatoform disorders in general practice: prevalence, functional impairment and comorbidity with anxiety and depressive disorders. $\mathrm{Br} \mathrm{J}$ Psychiatry. 2004;184:470-6.

19 Henningsen $\mathrm{P}$, Zimmermann T, Sattel $\mathrm{H}$. Medically unexplained physical symptoms, anxiety and depression: a meta-analytic review. Psychosom Med. 2003;65:528-33.

20 Henningsen $P$, Jakobsen T, Schiltenwolf M, Weiss MG. Somatization revisited: diagnosis and perceived causes of common mental disorders. J Nerv Ment Dis. 2005;193:85-92.

21 Tófoli LF, Andrade LH, Fortes S. Somatization in Latin America: a review on the classification of somatoform disorders, functional syndromes, and medically unexplained symptoms. Rev Bras Psiquiatr. 2011;33:S59-80.

22 Aaron LA, Buchwald D. A review of evidence for overlap among unexplained clinical conditions. Ann Intern Med. 2001;134:868-81.

23 Wessely S, Nimnuan C, Sharpe M. Functional somatic syndromes: one or many? Lancet. 1999;354:936-9.

24 Brasil MA, Appolinario JC, Fortes S. Functional somatic syndromes: many names for the same thing? In: Maj M, Akiskal H, Mezzich J. Somatoform disorders. Chicherter: John Wiley and Sons; 2005. WPA series Evidence and Experiences in Psychiatry.

25 Budtz-Lilly A, Schröder A, Rask MT, Fink P, Vestergaard M, Rosendal M. Bodily distress syndrome: a new diagnosis for functional disorders in primary care? BMC Fam Pract. 2015;16:180.

26 Fink $\mathrm{P}$, Schroder $\mathrm{A}$. One single diagnosis, bodily distress syndrome, succeeded to capture 10 diagnostic categories of functional somatic syndromes and somatoform disorders. J Psychosom Res. 2010;68: 415-26.

27 Fink P, Toft T, Hansen MS, Ornbol E, Olesen F. Symptoms and syndromes of bodily distress: an exploratory study of 978 internal medical, neurological, and primary care patients. Psychosom Med. 2007;69:30-9.

28 Fink $\mathrm{P}$, Rosendal M, Olesen $\mathrm{F}$. Classification of somatization and functional somatic symptoms in primary care. Aust NZ J Psychiatry. 2005;39:772-81.

29 Lam TP, Goldberg DP, Dowell AC, Fortes S, Mbatia JK, Minhas FA, et al. Proposed new diagnoses of anxious depression and bodily stress syndrome in ICD-11-PHC: an international focus group study. Fam Pract. 2013;30:76-87.

30 Goldberg DP, Reed GM, Robles R, Minhas F, Razzaque B, Fortes S, et al. Screening for anxiety, depression, and anxious depression in primary care: a field study for ICD-11 PHC. J Affect Disord. 2017;213: 199-206.

31 Goldberg DP, Reed GM, Robles R, Bobes J, Iglesias C, Fortes D, et al. Multiple somatic symptoms in primary care: a field study for ICD-11 PHC, WHO's revised classification of mental disorders in primary care settings. J Psychosom Res. 2016;91:48-54.

32 Lewis G, Pelosi AJ, Araya R, Dunn G. Measuring psychiatric disorder in the community: a standardized assessment for use by lay interviewers. Psychol Med. 1992;22:465-86.

33 Üstün B, Kostanjsek N, Chatterii S, Rehm J. Measuring health and disability: manual for WHO disability assessment schedule: WHODAS 2.0 [Internet]. 2010 [cited 2018 Feb 08]. apps.who.int/iris/bitstream/ 10665/43974/1/9789241547598_eng.pdf.

34 Escobar Jl. Cross-cultural aspects of the somatization trait. Hosp Community Psychiatry. 1987;38:174-80. 
35 Isaac M, Janca A, Burke KC, Costa e Silva JA, Acuda SW, Altamura $A C$, et al. Medically unexplained somatic symptoms in different cultures. A preliminary report from phase I of the World Health Organization International Study of somatoform disorders. Psychother Psychosom. 1995;64:88-93.

36 Muñoz RA, McBride ME, Brnabic AJ, López CJ, Hetem LA, Secin R, et al. Major depressive disorder in Latin America: the relationship between depression severity, painful somatic symptoms, and quality of life. J Affect Disord. 2005;86:93-8.
37 Goldberg DP, Prisciandaro JJ, Williams P. The primary health care version of ICD-11: the detection of common mental disorders in general medical settings. Gen Hosp Psychiatry. 2012;34:665-70.

38 Simon GE, VonKorff M, Piccinelli M, Fullerton C, Ormel J. An international study of the relation between somatic symptoms and depression. N Engl J Med. 1999;341:1329-35.

39 American Psychiatric Association. Diagnostic and Statistical Manual of Mental Disorders, Fifth Edition (DSM-5). Arlington: American Psychiatric Publishing; 2013. 